\title{
An Exploratory Study on the Acute Effects of Proprioceptive Exercise and/or Neuromuscular Taping on Balance Performance
}

\author{
Luca Russo, ${ }^{1}$ Paolo Bartolucci, ${ }^{1}$ Luca Paolo Ardigò, ${ }^{2,}$ " Johnny Padulo, ${ }^{3,4}$ Jelena Pausic, ${ }^{4}$ and Antonio \\ Dello Iacono ${ }^{5}$ \\ ${ }^{1}$ Department of Applied Clinical Sciences and Biotechnologies, University of L'Aquila, L'Aquila, Italy \\ ${ }^{2}$ Department of Neurosciences, School of Exercise and Sport Science, Biomedicine and Movement Sciences, University of Verona, Verona, Italy \\ ${ }^{3}$ University eCampus, Novedrate, Italy \\ ${ }^{4}$ Faculty of Kinesiology, University of Split, Split, Croatia \\ ${ }^{5}$ Zinman College of Physical Education and Sport Sciences, Wingate Institute, Netanya, Israel \\ "Corresponding author: Luca Paolo Ardigò, Department of Neurosciences, School of Exercise and Sport Science, Biomedicine and Movement Sciences, University of Verona, Via \\ Felice Casorati, 43, 37131, Verona, Italy. Tel: +39-3477266814, Fax: +39-0458425131, E-mail: luca.ardigo@univr.it
}

Received 2017 October 18; Revised 2017 December 03; Accepted 2018 March 10.

\begin{abstract}
Background: This study aimed at investigating the acute effects of combined EXERCISE and TAPING in comparison to isolated proprioceptive exercise (EXERCISE) and ankle neuromuscular taping (TAPING) on one - leg stability performance in rugby players.

Methods: Stability tests, performed on a stabilometric platform, were assessed for stability before and after above interventions. Performed stability tests were one - leg static stance (dominant leg and non - dominant leg) each with eyes open and eyes closed. The assessed dependent variables were: centre of pressure (CoP) path length; CoP speed; medio - lateral, and anterior - posterior sway. Sixteen male rugby players ( $27.3 \pm 3.3$ years; $177.3 \pm 7.3 \mathrm{~cm}$; $88.8 \pm 15.2 \mathrm{~kg})$ from a non - professional rugby team were tested in all above conditions, according to a cross - over study design.

Results: Most of investigated variables improved following EXERCISE + TAPING (CoP path length $-18.2 /-15.6 \%$, CoP speed -22.8/-17.7\%, and anterior-posterior sway -21.0/-16.3\%), in comparison with the other two protocols. EXERCISE + TAPING improved the stability control by combining the effects of both proprioceptive exercise and neuromuscular taping.

Conclusions: Such findings could suggest the benefits of planning long - term strategies using EXERCISE + TAPING protocols for improving the functional stability and for preventing re - occuring injuries.
\end{abstract}

Keywords: Ankle, Exercise, Proprioception

\section{Background}

Human postural demands and balance control are of primary interest for both daily life and athletic motion. The ability to manage one's own body in the environment is controlled by a complex interaction between musculoskeletal and neural system activities involving the reception and interpretation of information about the position of body parts, integration of sensorimotor inputs and execution of appropriate movement (1). Specifically for gait and balance tasks, ankle joint and foot structures are identified as great contributors to performance, given that they both represent the starting point from whence kinesthetic information arises and are the functional executors providing stability in response to the demanded skills (1). In fact, somatosensory inputs originate from three different receptors: pressure receptors in the skin, deep tendon sensory receptors, and joint receptors located in the distal part of the lower limb (2). In light of this background, the ankle and foot structures provide the propulsion for the whole body, absorb impact, and perform the functions of keeping the body upright against gravity and of maintaining posture during movement (3).

In the clinical field, changes in the control and the stability of the ankle are chronically obtained by means of proprioceptive exercises (4-7) and by applying both neuromuscular and rigid bandages to the joint $(5,8,9)$. Several types of exercise are proposed to improve proprioception, designed as either acute - designed strategies (6) or long - term training protocols. It has been widely reported that exercise groups involved in proprioceptive and neuromuscular training programs demonstrated significant improvement of passive and active ankle range of movement, better scores in most of the postural sway related variables and higher muscle activations combined with shorter re- 
action times of the muscles surrounding the ankle joint ( 5 , $6,10)$.

Neuromuscular taping (i.e., with adjustable elasticity) has been proposed as an effective alternative to the functional bandage for the passive stabilization and blockage (i.e., stiffness) of joints (8). Neuromuscular taping has a number of mechanical and neurophysiological advantages: it improves stability of the ankle joint and therefore has a positive effect on gait speed and balance ability in healthy adults (11) as well as positive effects on knee flexion range of movement, walking, and pain in patients with knee osteoarthritis (12). Neuromuscular taping has also the ability to control the centre of pressure (CoP) sway speed and lead to better performance in maintaining stability when a perturbation is applied (13). Studies have also shown that neuromuscular taping facilitates the neuromuscular reflexes (13). This capability is the result of the increase in sensory input caused by direct contact between the tape and the skin (14). MRI evidence indicates that neuromuscular taping affects both underlying and targeted tissues, as well as distant ones (15). In addition, some systematic reviews $(16,17)$ found anecdotal support for the usage of neuromuscular tape. Overall, other authors indicate the questionable potential advantages associated with its application. Williams et al. (17) concluded that there is little evidence to support the use of neuromuscular taping over other types of taping in the management or prevention of sports injuries and in improving strength and range of motion; but further studies are needed to confirm these findings. Serrao et al. (18) did not show any effect of neuromuscular taping on thigh muscles surface electromyography and perceived exertion during squat exercise in healthy subjects. The results of a meta - analysis (19) suggested that the effectiveness of neuromuscular taping is not muscle - group dependent. However, the reviewed studies investigated the potential effects of neuromuscular taping application on muscular outcomes but neglected the assessment of its well - known effects on postural control and joint stabilization. Indeed, both proprioceptive exercise and neuromuscular taping have been reported to enhance postural control by improving proprioception inputs; but investigations are lacking in the combined effects of these treatments, in order to select the best strategy to manage proprioception deficit.

\section{Objectives}

Taking into consideration the scientific evidence in the current literature, the main hypothesis of this study was that the concurrent application of neuromuscular taping combined with proprioceptive exercise - based protocols ("Taping Elastico Chinesiologico", T.E.CH. [i.e., "Elastic
Taping Applications in Kinesiology"] Method ${ }^{\circledR}$ ) would be more effective than the single strategy in inducing acute effects on balance ability. Therefore, this study compared the acute effects of using single or combined stabilization strategies on stability during a one - leg static balance task.

\section{Methods}

\subsection{Participants}

This study involved 16 male rugby players $(27.3 \pm 3.3$ years; $177.3 \pm 7.3 \mathrm{~cm}$; $88.8 \pm 15.2 \mathrm{~kg}$ [mean $\pm \mathrm{SD}$ ]), members of a non - professional rugby team. All players trained three days per week for $90 \mathrm{~min}$ a day, performing technical, tactical, strength, and speed training (plus match on Sunday). The three different experimental trials were administered on the same three days of week and at the same time of the day, in order to reduce possible bias and any potential effect of physical activities and diurnal variation. Players were asked to refrain from strenuous physical exercise during the $24 \mathrm{~h}$ before testing to limit residual effects due to previous effort. To identify which lower limb was the dominant one, the participants were asked to indicate their preferred kicking foot: $100 \%$ of the participants were identified as right - leg dominant. Written informed consent was obtained from the participants after they were given an oral explanation of the purpose, benefits, and potential risks of participating in the study. This study was approved by the institution's ethics committee in agreement with the Declaration of Helsinki.

The inclusion/exclusion criteria for the study participations were:

- To have participated in at least $90 \%$ of the training sessions with the same team.

- No history during the three months preceding the test of neuromuscular disease, vertigo, or any uncorrected visual problems.

- Absence of cardiovascular, neurologic, or pulmonary disease, balance problems, rheumatoid disease, or psychological disease.

- Absence of any kind of ankle injury or lower limb surgery.

- No use of sedative medication.

A randomized and crossover study design was used to compare the effects of using either combined or isolated strategies on subsequent stability performance. 


\subsection{Procedures}

Three experimental protocols were randomly administered on three different occasions. A subjects' block randomization in three groups was performed and the following protocols were administered:

- Protocol 1. Proprioceptive exercises and balance exercises performed on unstable surfaces (EXERCISE).

- Protocol 2. Neuromuscular tape(TAPING). Following the application of neuromuscular tape, subjects were free to move and walk, avoiding unstable surfaces, for about $25 \mathrm{~min}$.

- Protocol 3. Both neuromuscular tape and proprioceptive exercises on unstable surfaces (EXERCISE + TAPING) were applied/performed concurrently (i.e., taping was operated before proprioceptive exercises).

Upon each visit, the subjects performed a baseline stabilometric test (20), followed by one of the three conditions, and were then re - tested. The tests consisted of a one - leg static balance assessment lasting $10 \mathrm{sec}$ in the following conditions (3): alternate one - leg with open (OE) and closed (CE) eyes in a random sequence. The assessments were carried out in the same room and same conditions of light, temperature $\left(23^{\circ} \mathrm{C}\right)$, and humidity (18\%).

For the stabilometric assessment, a sensor matrix platform (FreeMed $40 \times 40$, Sensor Medica, Guidonia, Italy) with sampling rate of $>50 \mathrm{~Hz}$ (21) was used. The accuracy of the instrument was previously documented (22), while, for verifying the precision of the measures in our study, the stabilometric data recorded at each baseline point were compared. For each test, quantitative measurements of CoP path length, CoP path average speed, medio - lateral $(\Delta \mathrm{X})$ and anterior - posterior sway amplitude $(\Delta \mathrm{Y})$ were assessed (23).

The neuromuscular taping (Taping Elastico ${ }^{\circledR}$, ATS, Arezzo, Italy) was carried out according to the T.E.CH. Method ${ }^{\circledR}$ recommendations (Figure $1 \mathrm{~A}-\mathrm{C}$ ). Moreover, in all conditions the same operator operated the taping application, in order to avoid any bias effects due to inter-operator variability.

The proprioceptive exercise protocol was performed on different unstable surfaces that were placed in a series to create a compulsory path for the subjects. The exercise sequence had the same duration of the protocol described by Romero - Franco et al. $(6,22)$ : 25 min of proprioceptive exercise circuit with six balance exercises on unstable surfaces, lasting $30 \mathrm{~s}$ for each leg, with a $30 \mathrm{~s}$ recovery time, and repeated five times (Figure $2 \mathrm{~A}-\mathrm{F}$ ).
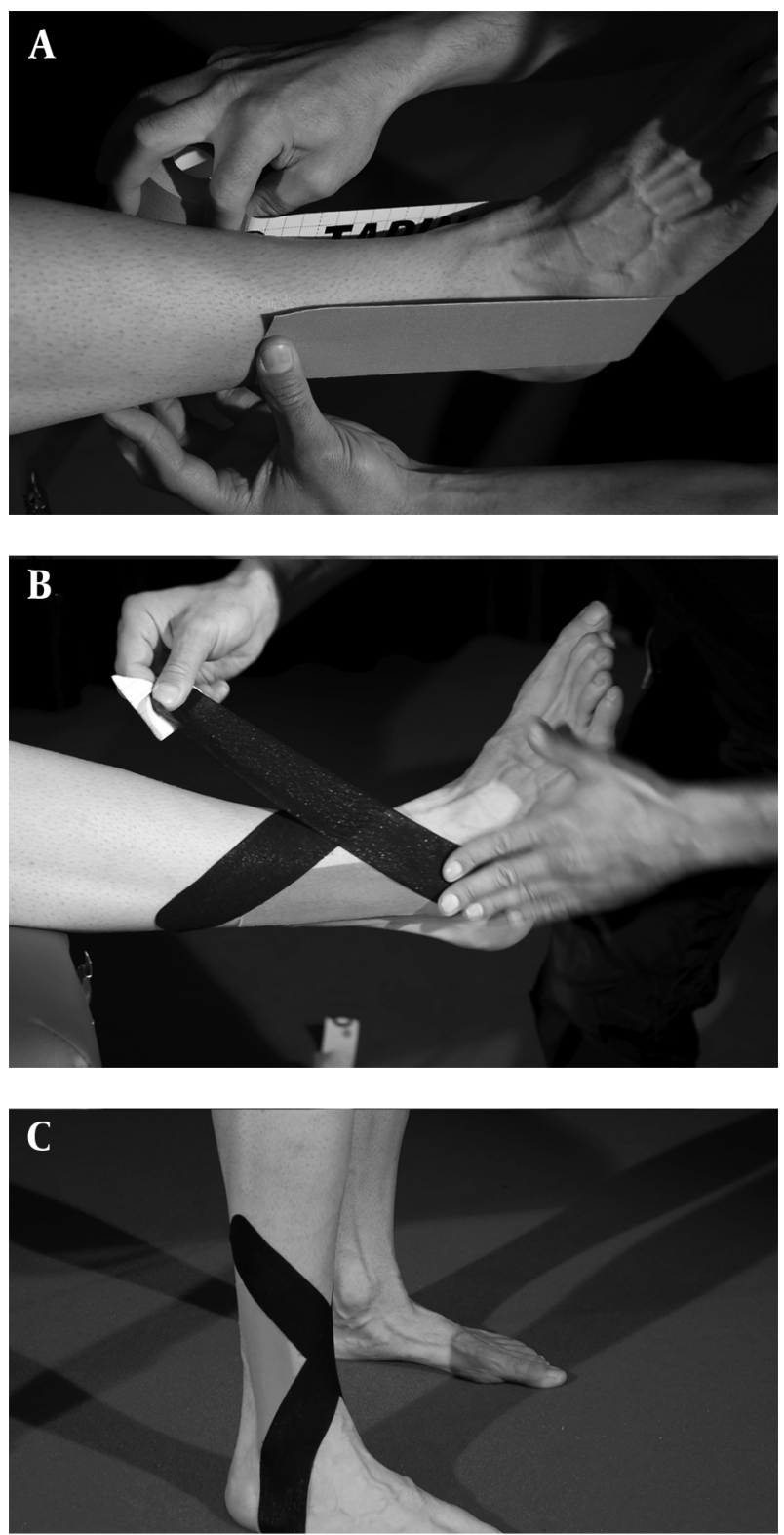

Figure 1. Placement of the Neuromuscular Tape on Subjects (A, B, and C).

\subsection{Statistical Analysis}

Descriptive statistics are presented as mean (SD, 95\% CI). Statistical analysis was performed by using SPSS software (version 21, SPSS Inc., Chicago, IL, USA). Assumption of data normality was verified by Kolmogorov - Smirnov test. A univariate ANOVA test with factor (each condition) post - hoc comparisons (LSD) was used to investigate differ- 

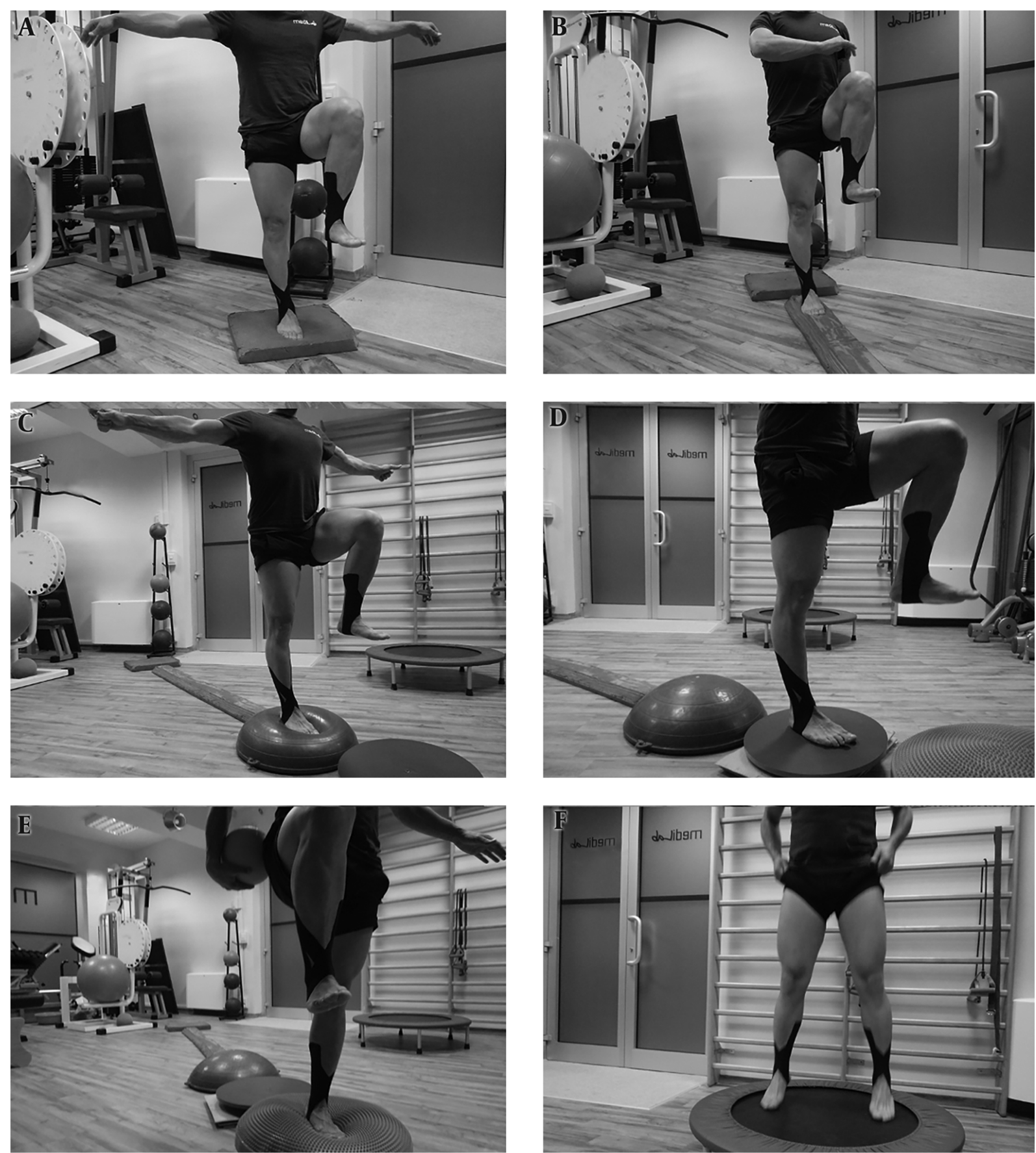

Figure 2. Proprioceptive exercise circuit's 6 workstations: (A) unstable foam mat in static stance, (B) unstable foam mat guide in dynamic stance, (C) air cushion in static stance, (D) stiff unstable tridimensional plate, (E) air cushion in lower - limb static stance and with ball movement around the trunk, (F) elastic trampoline with jump and stop task.

ences between the baseline measures of each testing day. Measures in the different conditions were analyzed by using a separate two - way ANOVA (condition $\mathrm{x}$ time) with re- peated measures and the paired $\mathrm{t}$ - test was used to compare pre- and post - treatment in the different conditions. The significance level was set at $\mathrm{P}<0.05$. In case of sig- 
nificance, Cohen's d effect size (ES) was calculated. The obtained ESs were shown and interpreted as proposed by Hopkins (www.sportsci.org/resource/stats) with ES $<0.2$ considered as trivial, 0.2-0.5 small, 0.6-1.1 moderate, 1.2-1.9 large and $>2$ very large.

\section{Results}

ANOVA showed no significant baseline stability performance differences between each testing day for all measurements in all conditions, with all $\mathrm{P}>0.05$ (Table 1), therefore type 1 error was excluded. Two - way ANOVA did not show any significant difference over conditions. The $t$ - test between pre- and post - values showed significant differences in effect of time (Tables 2 and 3 ).

For the non - dominant leg and OE condition, only following the EXERCISE + TAPING protocol, significant differences were found in the following CoP parameters: path length (-15.6\%, ES 0.7, moderate), average speed (-17.7\%, ES 0.8 , moderate), $\Delta \mathrm{X}$ and $\Delta \mathrm{Y}$ (-16.3 and ES 0.6, moderate; and $-26.4 \%$ and ES 0.9, moderate; respectively). Conversely, no significant changes were found for the dominant leg in $\mathrm{OE}$ condition task.

For the non - dominant leg and CE condition significant changes of the COP path length $(-18.2 \%$, ES 0.7, moderate), average speed (-22.8\%, ES 0.7 , moderate) and $\Delta \mathrm{Y}$ ($24.3 \%$, ES 0.7, moderate) occurred following the EXERCISE + TAPING protocol. For the dominant leg and CE condition COP path length and average speed were reduced by $-19.0 \%$ (ES 0.7, moderate) and -24.6\% (ES 0.8, moderate), respectively, following the TAPING application and COP path length, average speed and $\Delta \mathrm{Y}$ by $-14.0 \%$ (ES 0.7, moderate), $-18.5 \%$ (ES 0.7, moderate), and -18.5\% (ES 0.7, moderate), respectively, following the EXERCISE + TAPING protocol.

\section{Discussion}

Our findings clearly highlight that after performing EXERCISE + TAPING the stability performance improves in most of the assessment conditions. After applying TAPING, the stability performance improved only in the dominant leg and CE condition. After performing EXERCISE no significant stability performance change was found and this result does not conform to the findings of previous investigations (6) concerning the acute effect of isolated proprioceptive exercises on one-leg stability.

Despite the conflicting literature about the effects of neuromuscular tape on peripheral sensitivity and proprioception $(5,24,25)$, our results suggest that the application of a functional neuromuscular bandage, together with proprioceptive exercise, stimulate a greater activation of the local cutaneous receptors, with the role to provide unconscious afferent inputs, enhancing the stability and the joints' local control. Interestingly, these effects were greater in the CE conditions; therefore, it is reasonable that once the subjects were deprived of any visual feedback both sensorimotor and proprioceptive inputs would make a greater contribution in order to prevent impairments of the balance ability (26-28).

As hypothesized, EXERCISE + TAPING resulted in greater effects compared to the other experimental protocols in all conditions, except for dominant leg and OE. A previous study on the neurophysiological responses of muscle activity between dominant and non - dominant leg (29), showed inter - limb differences existing in the electrical activity of the peroneus longus during stability tasks. The authors concluded that due to the delayed activation, and consequently the lower contribution of the stabilizer muscle, the dominant limb is likely to be more susceptible to injury than the non - dominant one. Considering that the non-dominant leg represents the "support leg" mostly used during single - leg sport - specific tasks, the presence of beneficial effects of the EXERCISE + TAPING protocol may have occurred due to a good level of skillfulness in the non - dominant leg in our subjects (i.e., rugby players).

In our study, the combination of EXERCISE + TAPING induced effects also on both the dominant and the non - dominant leg with CE. The greater increased stability ability following the EXERCISE + TAPING protocol may have occurred due to an increased sensory onset and consequent activity induced by the stimulation caused by the combined use of proprioceptive exercises and neuromuscular tape; because when the visual feedback is lacking the contribution of sensorimotor, proprioceptive, and vestibular systems is required (30).

The evidence of potential positive effects on the receptor activation due to neuromuscular tape has not yet been specifically demonstrated through research (31), but experimental studies suggest that dynamic stability could be improved by the use of a functional bandage (32). Moreover, the use of taping, as a single method, for clinical use and for injury prevention strategies is still being questioned $(16,17$, 33).The potential combined effects of neuromuscular tape and other conventional methods (34) appear to be very interesting. We therefore suggest adding proprioceptive exercises to the taping application in order to gain both sensitivity of skin receptors and muscle proprioceptive stimulation.

Further studies with the aim of obtaining clearer evidence on the optimal training regimen (i.e., exercise modality) and training dose (i.e., volume and intensity) of exercises required for effective neuromuscular adaptations are needed. 


\begin{tabular}{|c|c|c|c|c|}
\hline Variable & CONDITION & EXERCISE & TAPING & EXERCISE + TAPING \\
\hline \multirow{2}{*}{ COP path length - non-dominant leg (mm) } & $\mathrm{OE}$ & $265.2(52.3,162.7-367.7)$ & $266.0(74.2,120.6-411.4)$ & $286.9(67.7,154.2-419.6)$ \\
\hline & $\mathrm{CE}$ & $742.5(125.0,497.5-987.5)$ & $762.3(265.1,242.7-1281.9)$ & $860.3(285.2,301.3-1419.3)$ \\
\hline \multirow{2}{*}{ COP path length - dominant leg (mm) } & $\mathrm{OE}$ & $429.1(61.8,308.0-550.2)$ & $423.4(78.2,270.1-567.7)$ & $442.7(81.8,282.4-603.0)$ \\
\hline & $\mathrm{CE}$ & $693.3(140.5,417.9-968.7)$ & $812.7(230.4,361.1-1264.3)$ & $806.5(172.7,468.0-1145.0)$ \\
\hline \multirow{2}{*}{ COP average speed - non-dominant leg $(\mathrm{mm} / \mathrm{s})$} & $\mathrm{OE}$ & $25.8(4.9,16.2-35.4)$ & $26.0(7.0,12.3-39.7)$ & $28.2(6.4,15.7-40.7)$ \\
\hline & $\mathrm{CE}$ & $57.8(11.9,34.5-81.1)$ & $60.2(25.3,10.6-109.8)$ & $69.1(28.5,13.2-125.0)$ \\
\hline \multirow{2}{*}{ COP average speed - dominant leg $(\mathrm{mm} / \mathrm{s})$} & $\mathrm{OE}$ & $26.7(5.9,15.1-38.3)$ & $25.3(7.5,10.6-40.0)$ & $27.7(9.5,9.1-46.3)$ \\
\hline & $\mathrm{CE}$ & $53.6(13.1,27.9-79.3)$ & $66.8(23.8,20.2-113.4)$ & $64.5(17.7,29.8-99.2)$ \\
\hline \multirow{2}{*}{ COP $\Delta X$ - non-dominant leg $(\mathrm{mm})$} & $\mathrm{OE}$ & $16.1(5.5,5.3-26.9)$ & $20.9(16.1,-10.7-52.5)$ & $18.9(5.2,8.7-29.1)$ \\
\hline & $\mathrm{CE}$ & $47.6(27.3,-5.9-101.1)$ & $58.7(45.7,-30.9-148.3)$ & $55.4(29.9,-3.2-114.0)$ \\
\hline \multirow{2}{*}{ COP $\Delta X$-dominant leg $(\mathrm{mm})$} & $\mathrm{OE}$ & $21.8(11.0,0.2-43.4)$ & $18.7(6.9,5.2-32.2)$ & $18.9(6.2,6.7-31.1)$ \\
\hline & $\mathrm{CE}$ & $44.6(18.5,8.3-80.9)$ & $62.7(40.8,-17.3-142.7)$ & $52.9(22.7,8.4-97.4)$ \\
\hline \multirow{2}{*}{ COP $\Delta \mathrm{Y}$ - non-dominant leg $(\mathrm{mm})$} & $\mathrm{OE}$ & $23.2(6.9,9.7-36.7)$ & $22.7(7.4,8.2-37.2)$ & $25.2(7.9,9.7-40.7)$ \\
\hline & $\mathrm{CE}$ & $53.1(19.5,14.9-91.3)$ & $56.6(36.1,-14.2-127.4)$ & $63.6(28.1,8.5-118.7)$ \\
\hline \multirow{2}{*}{ COP $\Delta Y$-dominant leg $(\mathrm{mm})$} & $\mathrm{OE}$ & $25.1(8.6-8.2-42.0)$ & $23.6(8.8,6.4-40.8)$ & $23.3(9.1,5.5-41.1)$ \\
\hline & $\mathrm{CE}$ & $54.4(20.1,15.0-93.8)$ & $66.8(31.4,5.3-128.3)$ & $62.1(18.0,26.8-97.4)$ \\
\hline
\end{tabular}

Abbreviations: COP, Centre of pressure; OE, open eyes; CE, closed eyes.

${ }^{\mathrm{a}}$ Data are presented as mean (SD, 95\% CI).

We acknowledge that there are some limitations in our study. Firstly, we cannot exclude biased effects due to eventual effects that previous proprioceptive training could have had on the following experimental sessions even those scheduled one or two weeks later. Secondly, according to the experimental trials-including the multiple one - leg stability task, some learning effects could have occurred which may have led to improved performances. However, the influence on the reported results could be likely limited by the use of a randomized order in the testing procedures and the lack of statistical differences for all the assessed measures between the three baseline time points. Therefore, our suggestions for future studies are: 1 ) to include a greater sample size and 2) to design long-term training interventions for investigating both the chronic effects of similar protocols and the eventual residual effects following detraining phases by multiple time-point assessments.

\subsection{Conclusion}

Our results provide preliminary practical evidence in suggesting the combined use of proprioceptive exercise and neuromuscular tape for obtaining greater effects in terms of stabilization in the one - leg standing position. We suggest that it may be beneficial to perform exercises while wearing neuromuscular tape in order to exploit all the perceptual and sensitive effects possible, and, in turn, to maximize control of the body segments and the whole body balance.

\section{Acknowledgments}

The authors wish to thank all the players who volunteered within this study and Ms. Dinah Olswang for English editing. 


\begin{tabular}{|c|c|c|c|c|c|c|c|c|c|}
\hline \multirow[b]{2}{*}{ Protocol } & \multirow[b]{2}{*}{ Leg } & \multirow[b]{2}{*}{ Pre } & \multirow[b]{2}{*}{ Post } & \multirow[b]{2}{*}{ Av Diff \% } & \multirow[b]{2}{*}{ ES } & \multicolumn{2}{|c|}{ Group Interaction } & \multicolumn{2}{|c|}{ Time $x$ Condition } \\
\hline & & & & & & $\mathbf{F}$ & $\mathbf{P}$ & $\mathbf{F}$ & $\mathbf{P}$ \\
\hline \multicolumn{10}{|c|}{ COP Path Length $(\mathrm{mm})$} \\
\hline EXERCISE & ND & $265.2(52.3)$ & $260.3(67.7)$ & -1.9 & 0.1 & 0.090 & 0.914 & 1.888 & 0.163 \\
\hline TAPING & & $266.0(74.2)$ & $275.4(88.6)$ & 3.6 & 0.1 & & & & \\
\hline EXERCISE + TAPING & & $286.9(67.7)$ & $242.3(56.5)$ & $-15.6^{\mathrm{a}}$ & 0.7 & & & & \\
\hline \multicolumn{10}{|c|}{ COP Average Speed $(\mathrm{mm} / \mathrm{s})$} \\
\hline EXERCISE & ND & $25.8(4.9)$ & $24.3(5.9)$ & -5.9 & 0.3 & 0.073 & 0.929 & 1.256 & 0.295 \\
\hline TAPING & & $26.0(7.0)$ & $24.6(6.1)$ & -5.5 & 0.2 & & & & \\
\hline EXERCISE + TAPING & & $28.2(6.4)$ & $23.2(5.8)$ & $-17.7^{\mathrm{a}}$ & 0.8 & & & & \\
\hline \multicolumn{10}{|c|}{$\operatorname{COP} \Delta \mathrm{X}(\mathrm{mm})$} \\
\hline EXERCISE & ND & $16.1(5.5)$ & $17.1(7.3)$ & 6.3 & 0.2 & 2.003 & 0.147 & 0.409 & 0.667 \\
\hline TAPING & & $20.9(16.1)$ & $21.1(12.0)$ & 0.8 & 0.0 & & & & \\
\hline EXERCISE + TAPING & & $18.9(5.2)$ & $15.8(5.1)$ & $-16.3^{\mathrm{a}}$ & 0.6 & & & & \\
\hline \multicolumn{10}{|c|}{$\operatorname{COP} \Delta \mathrm{Y}(\mathrm{mm})$} \\
\hline EXERCISE & ND & $23.2(6.9)$ & $22.5(11.3)$ & -3.1 & 0.1 & 1.381 & 0.262 & 2.422 & 0.100 \\
\hline TAPING & & $22.9(7.4)$ & $29.2(20.3)$ & 27.6 & 0.4 & & & & \\
\hline EXERCISE + TAPING & & $25.2(7.9)$ & $18.6(6.7)$ & $-26.4^{\mathrm{a}}$ & 0.9 & & & & \\
\hline \multicolumn{10}{|c|}{ COP Path Length (mm) } \\
\hline EXERCISE & $\mathrm{D}$ & $429.1(61.8)$ & $415.7(73.1)$ & -3.1 & 0.2 & 0.256 & 0.775 & 0.072 & 0.931 \\
\hline TAPING & & $423.4(78.2)$ & $406.0(74.9)$ & -4.1 & 0.2 & & & & \\
\hline EXERCISE + TAPING & & $442.7(81.8)$ & $420.2(76.7)$ & -5.1 & 0.3 & & & & \\
\hline \multicolumn{10}{|c|}{ COP Average Speed $(\mathrm{mm} / \mathrm{s})$} \\
\hline EXERCISE & $\mathrm{D}$ & $26.7(5.9)$ & $25.4(7.0)$ & -5.1 & 0.2 & 0.286 & 0.752 & 0.259 & 0.773 \\
\hline TAPING & & $25.3(7.5)$ & $24.1(6.2)$ & -4.8 & 0.2 & & & & \\
\hline EXERCISE + TAPING & & $27.7(9.5)$ & $24.9(7.5)$ & -10.1 & 0.3 & & & & \\
\hline \multicolumn{10}{|c|}{$\operatorname{COP} \Delta \mathrm{X}(\mathrm{mm})$} \\
\hline EXERCISE & $\mathrm{D}$ & $21.8(11.0)$ & $19.1(7.1)$ & -12.1 & 0.3 & 0.578 & 0.565 & 0.291 & 0.749 \\
\hline TAPING & & $18.7(6.9)$ & $18.4(6.2)$ & -1.8 & 0.0 & & & & \\
\hline EXERCISE + TAPING & & $18.9(6.2)$ & $18.3(6.0)$ & -3.0 & 0.1 & & & & \\
\hline \multicolumn{10}{|c|}{$\operatorname{COP} \Delta \mathrm{Y}(\mathbf{m m})$} \\
\hline EXERCISE & $\mathrm{D}$ & $25.1(8.6)$ & $20.6(6.3)$ & -17.7 & 0.6 & 0.005 & 0.995 & 0.418 & 0.661 \\
\hline TAPING & & $23.6(8.8)$ & $21.6(8.1)$ & -8.4 & 0.2 & & & & \\
\hline EXERCISE + TAPING & & $23.3(9.1)$ & $22.1(7.7)$ & -5.2 & 0.1 & & & & \\
\hline
\end{tabular}

Abbreviations; COP, centre of pressure; ND, not dominant leg; D, dominant leg. ${ }^{a}$ Pre - Post Protocol Values Significant Difference. 


\begin{tabular}{|c|c|c|c|c|c|c|c|c|c|}
\hline \multirow[b]{2}{*}{ Protocol } & \multirow[b]{2}{*}{ Leg } & \multirow[b]{2}{*}{ Pre } & \multirow[b]{2}{*}{ Post } & \multirow[b]{2}{*}{ Av Diff \% } & \multirow[b]{2}{*}{ ES } & \multicolumn{2}{|c|}{ Group Interaction } & \multicolumn{2}{|c|}{ Time $x$ Condition } \\
\hline & & & & & & $\mathbf{F}$ & $\mathbf{P}$ & $\mathbf{F}$ & $\mathbf{P}$ \\
\hline \multicolumn{10}{|c|}{ COP Path Length (mm) } \\
\hline EXERCISE & ND & $742.5(125.0)$ & $714.9(124.5)$ & -3.7 & 0.2 & 0.414 & 0.663 & 0.983 & 0.382 \\
\hline TAPING & & $762.3(265.1)$ & $726.0(297.4)$ & -4.8 & 0.1 & & & & \\
\hline EXERCISE+TAPING & & $860.3(285.2)$ & $703.6(172.2)$ & $-18.2^{\mathrm{a}}$ & 0.7 & & & & \\
\hline \multicolumn{10}{|c|}{ COP Average Speed $(\mathrm{mm} / \mathrm{s})$} \\
\hline EXERCISE & ND & $57.8(11.9)$ & $54.6(12.4)$ & -5.5 & 0.3 & 0.371 & 0.692 & 0.951 & 0.394 \\
\hline TAPING & & $60.2(25.3)$ & $55.7(29.1)$ & -7.4 & 0.2 & & & & \\
\hline EXERCISE+TAPING & & $69.1(28.5)$ & $53.3(17.4)$ & $-22.8^{\mathrm{a}}$ & 0.7 & & & & \\
\hline \multicolumn{10}{|c|}{$\operatorname{COP} \Delta \mathrm{X}(\mathrm{mm})$} \\
\hline EXERCISE & ND & $47.6(27.3)$ & $46.2(15.9)$ & -2.9 & 0.1 & 2.074 & 0.137 & 0.756 & 0.475 \\
\hline TAPING & & $58.7(45.7)$ & $72.7(73.2)$ & 23.8 & 0.2 & & & & \\
\hline EXERCISE+TAPING & & $55.4(29.9)$ & $43.8(26.2)$ & -21.0 & 0.6 & & & & \\
\hline \multicolumn{10}{|c|}{$\operatorname{COP} \Delta \mathrm{Y}(\mathbf{m m})$} \\
\hline EXERCISE & ND & $53.1(19.5)$ & $49.8(20.4)$ & -6.2 & 0.2 & 0.343 & 0.711 & 0.877 & 0.423 \\
\hline TAPING & & $56.6(36.1)$ & $61.0(57.2)$ & 7.8 & 0.1 & & & & \\
\hline EXERCISE+TAPING & & $63.6(28.1)$ & $48.1(18.1)$ & $-24.3^{\mathrm{a}}$ & 0.7 & & & & \\
\hline \multicolumn{10}{|c|}{ COP Path Length (mm) } \\
\hline EXERCISE & $\mathrm{D}$ & $693.3(140.5)$ & $756.0(242.1)$ & 9.0 & 0.3 & 0.090 & 0.914 & 6.812 & 0.003 \\
\hline TAPING & & $812.7(230.4)$ & $658.6(183.9)$ & $-19.0^{\mathrm{a}}$ & 0.7 & & & & \\
\hline EXERCISE+TAPING & & $806.5(172.7)$ & $693.8(160.0)$ & $-14.0^{\mathrm{a}}$ & 0.7 & & & & \\
\hline \multicolumn{10}{|c|}{ COP Average Speed $(\mathrm{mm} / \mathrm{s})$} \\
\hline EXERCISE & $\mathrm{D}$ & $53.6(13.1)$ & $58.9(24.3)$ & 9.9 & 0.3 & 0.097 & 0.908 & 6.642 & 0.003 \\
\hline TAPING & & $66.8(23.8)$ & $50.3(18.3)$ & $-24.6^{\mathrm{a}}$ & 0.8 & & & & \\
\hline EXERCISE+TAPING & & $64.5(17.7)$ & $52.6(15.8)$ & $-18.5^{\mathrm{a}}$ & 0.7 & & & & \\
\hline \multicolumn{10}{|c|}{$\operatorname{COP} \Delta \mathrm{X}(\mathrm{mm})$} \\
\hline EXERCISE & $\mathrm{D}$ & $44.6(19.5)$ & $46.3(24.3)$ & 4.0 & 0.1 & 0.582 & 0.563 & 1.344 & 0.271 \\
\hline TAPING & & $62.7(40.8)$ & $45.4(35.8)$ & -27.6 & 0.5 & & & & \\
\hline EXERCISE+TAPING & & $52.9(22.7)$ & $44.2(17.4)$ & -16.4 & 0.4 & & & & \\
\hline \multicolumn{10}{|c|}{$\operatorname{COP} \Delta \mathrm{Y}(\mathbf{m m})$} \\
\hline EXERCISE & $\mathrm{D}$ & $54.4(20.1)$ & $66.6(42.2)$ & 22.5 & 0.4 & 0.149 & 0.862 & 2.629 & 0.83 \\
\hline TAPING & & $66.8(31.4)$ & $52.2(35.6)$ & -21.8 & 0.4 & & & & \\
\hline EXERCISE+TAPING & & $62.1(18.0)$ & $50.6(17.2)$ & $-18.5^{\mathrm{a}}$ & 0.7 & & & & \\
\hline
\end{tabular}

Abbreviations; COP, centre of pressure; ND, not dominant leg; D, dominant leg.

${ }^{\mathrm{a}}$ Pre - post protocol values significant difference.

\section{Footnotes}

Conflict of Interest: L. Russo and P. Bartolucci own T.E.CH. Method $^{\circledR}$. L. P. Ardigò, J. Padulo, J. Pausic, and A. Dello Iacono do not have any conflict of interest in this paper.

Funding/Support: No external financial support has been received.

\section{References}

1. Shumway-Cook A, Woollacott MH. Motor Control: Theory and Practical
Applications. Baltimore (MD): Williams \& Wilkins; 1995.

2. Ghez C. Posture. In: Kandel ER, Schwartz JH, Jessel TM, editors. Principles of neural science. New York: Elsevier; 1991. p. 596-607.

3. Winter DA, Patla AE, Rietdyk S, Ishac MG. Ankle muscle stiffness in the control of balance during quiet standing. J Neurophysiol. 2001;85(6):2630-3. doi: 10.1152/jn.2001.85.6.2630. [PubMed: 11387407].

4. Freeman MA, Dean MR, Hanham IW. The etiology and prevention of functional instability of the foot. J Bone Joint Surg Br. 1965;47(4):67885. [PubMed: 5846767].

5. Hughes T, Rochester P. The effects of proprioceptive exercise and taping on proprioception in subjects with functional ankle instability: a review of the literature. Phys Ther Sport. 2008;9(3):136-47. doi: 10.1016/j.ptsp.2008.06.003. [PubMed: 19083714]. 
6. Romero-Franco N, Martinez-Amat A, Hita-Contreras F, Martinez-Lopez EJ. Short-term Effects of a Proprioceptive Training Session with Unstable Platforms on the Monopodal Stabilometry of Athletes. J Phys Ther Sci. 2014;26(1):45-51. doi: 10.1589/jpts.26.45. [PubMed: 24567674]. [PubMed Central: PMC3927040].

7. Tropp H, Ekstrand J, Gillquist J. Stabilometry in functional instability of the ankle and its value in predicting injury. Med Sci Sports Exerc. 1984;16(1):64-6. [PubMed: 6708781].

8. Bicici S, Karatas N, Baltaci G. Effect of athletic taping and kinesiotaping( $\mathrm{R})$ on measurements of functional performance in basketball players with chronic inversion ankle sprains. Int J Sports Phys Ther. 2012;7(2):154-66. [PubMed: 22530190]. [PubMed Central: PMC3325641].

9. Simon J, Donahue M. Effect of ankle taping or bracing on creating an increased sense of confidence, stability, and reassurance when performing a dynamic-balance task. J Sport Rehabil. 2013;22(3):229-33. [PubMed: 23579444].

10. Romero-Franco N, Martinez-Lopez E, Lomas-Vega R, Hita-Contreras F, Martinez-Amat A. Effects of proprioceptive training program on core stability and center of gravity control in sprinters. J Strength Cond Res. 2012;26(8):2071-7. doi: 10.1519/JSC.0b013e31823b06e6. [PubMed: 21997455].

11. Kim MK, Cha HG. The effects of ankle joint taping on gait and balance ability of healthy adults. J Phys Ther Sci. 2015;27(9):29134. doi: 10.1589/jpts.27.2913. [PubMed: 26504323]. [PubMed Central: PMC4616124].

12. Kaya Mutlu E, Mustafaoglu R, Birinci T, Razak Ozdincler A. Does Kinesio Taping of the Knee Improve Pain and Functionality in Patients with Knee Osteoarthritis?: A Randomized Controlled Clinical Trial. Am J Phys Med Rehabil. 2017;96(1):25-33. doi: 10.1097/PHM.0000000000000520. [PubMed: 27149590].

13. Wilkerson GB. Biomechanical and Neuromuscular Effects of Ankle Taping and Bracing. J Athl Train. 2002;37(4):436-45. [PubMed: 12937565]. [PubMed Central: PMC164375].

14. Atc BT. The Effects of Prophylactic Taping on Ankle Joint Motion and Performance. Legacy ETDs. 1996;491.

15. Pamuk U, Yucesoy CA. MRI analyses show that kinesio taping affects much more than just the targeted superficial tissues and causes heterogeneous deformations within the whole limb. J Biomech. 2015;48(16):4262-70. doi: 10.1016/j.jbiomech.2015.10.036. [PubMed: 26556717].

16. Kalron A, Bar-Sela S. A systematic review of the effectiveness of Kinesio Taping-fact or fashion? Eur J Phys Rehabil Med. 2013;49(5):699-709. [PubMed: 23558699].

17. Williams S, Whatman C, Hume PA, Sheerin K. Kinesio taping in treatment and prevention of sports injuries: a meta-analysis of the evidence for its effectiveness. Sports Med. 2012;42(2):153-64. doi: 10.2165/11594960-000000000-00000. [PubMed: 22124445].

18. Serrao JC, Mezencio B, Claudino JG, Soncin R, Miyashiro PL, Sousa EP, et al. Effect of 3 Different Applications of Kinesio Taping Denko(R) on Electromyographic Activity: Inhibition or Facilitation of the Quadriceps of Males During Squat Exercise. J Sports Sci Med. 2016;15(3):403-9. [PubMed: 27803618]. [PubMed Central: PMC4974852].

19. Csapo R, Alegre LM. Effects of Kinesio((R)) taping on skeletal muscle strength-A meta-analysis of current evidence. J Sci Med Sport. 2015;18(4):450-6. doi: 10.1016/j.jsams.2014.06.014. [PubMed: 25027771].

20. Russo L, D’Eramo U, Padulo J, Foti C, Schiffer R, Scoppa F. Day- time effect on postural stability in young sportsmen. Muscles Ligaments Tendons J. 2015;5(1):38-42. [PubMed: 25878986]. [PubMed Central: PMC4396675].

21. Scoppa F, Capra R, Gallamini M, Shiffer R. Clinical stabilometry standardization: basic definitions-acquisition intervalsampling frequency. Gait Posture. 2013;37(2):290-2. doi: 10.1016/j.gaitpost.2012.07.009. [PubMed: 22889928].

22. Romero-Franco N, Martinez-Lopez EJ, Lomas-Vega R, HitaContreras F, Osuna-Perez MC, Martinez-Amat A. Short-term effects of proprioceptive training with unstable platform on athletes' stabilometry. J Strength Cond Res. 2013;27(8):2189-97. doi: 10.1519/JSC.ob013e31827de04c. [PubMed: 23207893].

23. Vando S, Unim B, Cassarino S, Paulo J, Masala D. Effectiveness of perceptual training-proprioceptive feedback in a virtual visual diverse group of healthy subjects: a pilot study. Epidemiol Biostatistics Publ Health. 2013;10(2).

24. Halseth T, McChesney JW, Debeliso M, Vaughn R, Lien J. The effects of kinesio taping on proprioception at the ankle. J Sports Sci Med. 2004;3(1):1-7. [PubMed: 24497814]. [PubMed Central: PMC3896108].

25. Spanos S, Brunswic M, Billis E. The effect of taping on the proprioception of the ankle in a non-weight bearing position, amongst injured athletes. The Foot. 2008;18(1):25-33. doi:10.1016/j.foot.2007.07.003.

26. Gagey PM, Weber BP. Posturologie. Régulation et dérèglements de la station debout. Paris: Masson; 2005. French.

27. Nakagawa H, Ohashi N, Watanabe Y, Mizukoshi K. The contribution of proprioception to posture control in normal subjects. Acta Otolaryngol Suppl.1993;504:112-6. [PubMed: 8470514].

28. Yasuda T, Nakagawa T, Inoue H, Iwamoto M, Inokuchi A. The role of the labyrinth, proprioception and plantar mechanosensors in the maintenance of an upright posture. Eur Arch Otorhinolaryngol. 1999;256 Suppl 1:S27-32. [PubMed:10337523].

29. Knight AC, Weimar WH. Difference in response latency of the peroneus longus between the dominant and nondominant legs.J Sport Rehabil. 2011;20(3):321-32. [PubMed: 21828384].

30. Chaouachi A, Padulo J, Kasmi S, Othmen AB, Chatra M, Behm DG. Unilateral static and dynamic hamstrings stretching increases contralateral hip flexion range of motion. Clin Physiol Funct Imaging. 2017;37(1):23-9. doi: 10.1111/cpf.12263. [PubMed: 26017182].

31. Nakajima MA, Baldridge $C$. The effect of kinesio(R) tape on vertical jump and dynamic postural control. Int J Sports Phys Ther. 2013;8(4):393-406. [PubMed: 24175126]. [PubMed Central: PMC3812836].

32. Gomez-Soriano J, Abian-Vicen J, Aparicio-Garcia C, Ruiz-Lazaro P, Simon-Martinez C, Bravo-Esteban E, et al. The effects of Kinesio taping on muscle tone in healthy subjects: a double-blind, placebocontrolled crossover trial. Man Ther. 2014;19(2):131-6. [PubMed: 24829961].

33. Parreira Pdo C, Costa Lda C, Hespanhol LJ, Lopes AD, Costa LO. Current evidence does not support the use of Kinesio Taping in clinical practice: a systematic review. J Physiother. 2014;60(1):31-9. doi: 10.1016/j.jphys.2013.12.008. [PubMed: 24856938].

34. Added MA, Costa LO, Fukuda TY, de Freitas DG, Salomao EC, Monteiro $\mathrm{RL}$, et al. Efficacy of adding the Kinesio Taping method to guidelineendorsed conventional physiotherapy in patients with chronic nonspecific low back pain: a randomised controlled trial. BMC Musculoskelet Disord. 2013;14:301. doi: 10.1186/1471-2474-14-301. [PubMed: 24156687]. [PubMed Central: PMC3870967]. 